\title{
Incidence of acute mountain sickness in young adults at 3200 meters: comparison of the Lake Louise Scoring and Chinese Scoring Systems
}

G.Z. Chen, J. Qin, J. Yu, X.B. Gao, J.Q. Dong, W. Lu, S.Z. Bian, Y. Zeng and $\mathrm{L}$. Huang

Institute of Cardiovascular Diseases of PLA, Xinqiao Hospital, Third Military Medical University, Chongqing, China

Corresponding author: L. Huang

E-mail: lanhuangcn@163.com

Genet. Mol. Res. 12 (4): 6790-6801 (2013)

Received May 22, 2013

Accepted October 2, 2013

Published December 16, 2013

DOI http://dx.doi.org/10.4238/2013.December.16.5

\begin{abstract}
The purpose of this study was to compare two scoring systems used for the diagnosis of acute mountain sickness (AMS): the Lake Louise Scoring (AMS-LLS) and the Chinese Scoring Systems (AMS-CSS). In total, 339 healthy young adult volunteers residing at sea level ascended to $3200 \mathrm{~m}$ by train and bus over a total journey time of $48 \mathrm{~h}$. All subjects ascended in the same manner and were divided into three groups that were assessed after one $(\mathrm{N}=88)$, two $(\mathrm{N}=91)$, and three $(\mathrm{N}=160)$ nights, respectively, at altitude. The overall incidence of AMS was $17.11 \%(\mathrm{~N}=58)$ and $29.79 \%(\mathrm{~N}=101)$ according to the AMS-LLS and AMS-CSS, respectively. Two participants $(0.59 \%)$ experienced high-altitude pulmonary edema. Both scoring systems showed the highest incidence of AMS after the second night at high altitude. The AMS-CSS and AMS-LLS scores were significantly correlated (Pearson's $r=0.820, \mathrm{P}<0.001$ ). The AMS-CSS identified all AMS subjects diagnosed by the AMS-LLS, and an additional 43 subjects. The dominant symptoms were reduced exercise tolerance $(61.7 \%)$, fatigue $(49.0 \%)$, dizziness $(28.9 \%)$, chest distress $(28.3 \%)$,
\end{abstract}


and headache (27.4\%). Compared with the AMS-LLS, the sensitivity, specificity, and positive and negative predictive values of the AMSCSS were 100, 84.7, 57.43, and 100\%, respectively. There was no relationship between oxygen saturation levels and AMS scores at 3200 m. In summary, the AMS-CSS was similar to AMS-LLS, except that it resulted in more positive diagnoses, and headache did not play a large diagnostic role.

Key words: Acute mountain sickness; Acute hypoxia; Pulse oximetry; Tibetan plateau; Headache

\section{INTRODUCTION}

Rapid ascent to altitudes above $2500 \mathrm{~m}$ is associated with a risk of developing acute mountain sickness (AMS), high-altitude cerebral edema (HACE), or high-altitude pulmonary edema (HAPE) (Luks et al., 2010). AMS not only impairs human health, but also influences the economic costs of high-altitude travel. AMS is a potential issue for hikers, and it may also be harmful to those engaged in disaster relief work at high altitudes. Following an earthquake in Yushu County (3980 m), Qinghai Province, China, on April 14, 2010, 28 of 32 rescuers (81.25\%) suffered from AMS (Jiao et al., 2010). Of the approximately 20,000 rescuers involved in the aftermath of this earthquake (one of the highest that has occurred), the incidence of HAPE was $0.73 \%(\mathrm{Wu}, 2011)$. As a result, many healthcare providers now recognize the importance of the prevention and treatment of AMS. The incidences of AMS reported have varied substantially due to differences between studies in altitude, ascent rate, previous exposure to high altitude, and individual susceptibility (Schneider et al., 2002; Vardy et al., 2006; Wu et al., 2009; Karinen et al., 2010; Kayser et al., 2010; Newcomb et al., 2011). The Lake Louise Scoring System (AMS-LLS), which depends on the subject's personal appreciation of the severity of symptoms, is commonly used to diagnose AMS (Roach et al., 1993). The search for a more objective set of criteria has received some attention, but further research is clearly needed (Kayser et al., 2010). Unfortunately, the pathogenesis of AMS is not yet fully understood despite the fact that it has been frequently studied. However, these trials were small, and the numbers of participants in the trials were small, with sample sizes often less than 100. Because of the harsh environment at high altitudes, there is also a lack of large, randomized clinical trials assessing the prevention and treatment of AMS. This makes it extremely difficult to study the incidence of AMS with any degree of precision.

It is well known that headache is a cornerstone symptom for the diagnosis of AMS, and that this may be associated with the trigeminovascular system (Sanchez del Rio and Moskowitz, 1999). There is, however, considerable controversy regarding the role of headache in AMS (Roach et al., 2011; West, 2011). Ren et al. (2000) reported that the incidence of AMS was $57.2 \%$ in unacclimatized healthy men at an altitude of $3600 \mathrm{~m}$, and emphasized that headache was not essential according to the Chinese Scoring System for AMS (AMSCSS), which is widely used by Chinese researchers [see also, Third Ad Hoc Committee on High Altitude Illnesses of Chinese Medical Association (1996) and West (2010)]. China is one of the largest high-altitude countries in the world. Many people in China live, travel, and 
work at high altitudes. Extensive scientific research has been performed to investigate AMS in China, and numerous articles have been published concerning the use of the AMS-CSS in the diagnosis of this disease. However, there are certain differences between the AMS-CSS and AMS-LLS, and language discrepancies can further impair the comparison of AMS data between China and Western countries. Direct comparisons of these two scoring systems, used at the same time in the same cohort, have yet to be reported. Therefore, we designed the present study to determine: 1) the incidence of AMS, as defined by the AMS-LLS and AMSCSS in the same cohort of subjects, and 2) the role of headache in the diagnosis of AMS.

\section{MATERIAL AND METHODS}

\section{Questionnaire and scoring systems}

We designed a questionnaire that included both the AMS-LLS and the AMS-CSS. In brief, we investigated the five main symptoms that underlie the AMS-LLS questionnaire, including headache, gastrointestinal (GI) upset, fatigue, dizziness, and insomnia. We also documented other symptoms, such as palpitations, shortness of breath, chest distress, poor appetite, dyspnea, tinnitus, abdominal distention, constipation, diarrhea, cough, and decreased exercise tolerance. Based on the AMS-LLS, all subjects diagnosed with AMS had headaches and at least one of the following symptoms: GI upset, fatigue, dizziness, or insomnia. An AMS-LLS score of $\geq 3$ points established the diagnosis of AMS, while a score of $\geq 5$ points defined severe AMS (Table 1). The AMS-CSS values are shown in Tables 2 and 3 (West, 2010). The parameters used to calculate these scores were headache, GI upset (nausea, vomiting), abdominal distention, diarrhea, constipation, shortness of breath, chest distress, palpitations, insomnia, lethargy, dizziness, blurred vision, and paresthesia. Using the AMS-CSS, the diagnosis of AMS was made if the patient had headache, vomiting, or a total score of $\geq 5$ points. Details of the AMS grades, as determined using AMS-CSS, are shown in Table 2.

Table 1. Assessment of the degree and score of acute mountain sickness-Lake Louise Scoring System (AMS-LLS).

\begin{tabular}{lccccc}
\hline & Headache & Gastrointestinal $(\mathrm{GI})$ upset & Fatigue & Dizziness & Insomnia \\
\hline No symptom & 0 & 0 & 0 & 0 & 1 \\
Mild & 1 & 1 & 1 & 2 & 1 \\
Moderate & 2 & 2 & 2 & 3 & 2 \\
Severe & 3 & 3 & 3 & 3 \\
\hline
\end{tabular}

All subjects diagnosed with AMS had headaches and at least one of the following symptoms: GI upset, fatigue, dizziness, or insomnia. AMS-LLS score of $\geq 3$ points = AMS; AMS-LLS score of 3-4 points = mild AMS; AMSLLS score of $\geq 5$ points $=$ severe AMS.

Table 2. Assessment of the severity and grade of AMS-CSS.

\begin{tabular}{ll}
\hline Degree & Score/grade \\
\hline Normal $( \pm)$ & Total score of 1-4 points \\
Mild $(+)$ & Headache,+ or vomiting + ; or total score of $5-10$ points \\
Moderate $(++)$ & Headache,++ or vomiting ++ ; or total score of $11-15$ points \\
Severe $(+++)$ & Headache,+++ or vomiting +++ ; or total score of $\geq 16$ points \\
\hline
\end{tabular}




\begin{tabular}{lc} 
Table 3. Assessment of the degree and score of AMS-CSS. & Degree \\
\hline Symptoms & Score \\
\hline Headache & + \\
1. No headache, no suffering expression, no effect on daily activity. & 1 \\
2. Mild headache with suffering expression; obvious improvement & + \\
of headache after taking regular analgesic medicine; no effect on daily activity. & + \\
3. Moderate headache with suffering expression; slight improvement of headache & ++ \\
after taking regular analgesic medicine; daily activity is affected. & + \\
4. Severe and unbearable headache; lie in bed and cannot get up; no effect of regular analgesic medication. & + \\
Vomiting & ++ \\
1. Vomiting 1 to 2 times a day; vomit contains only intake food; obvious improvement & + \\
with regular anti-vomiting medication; no effect on daily activity. & + \\
2. Vomiting 3 to 4 times a day; final vomit contains gastric juice; slight improvement & ++ \\
with anti-vomiting medication; daily activity is affected. & + \\
3. Vomiting more than 5 times a day; must lie in bed and cannot get up; & 4 \\
no improvement with regular anti-vomiting medication. & 7 \\
Other & + \\
Dizziness/light-headedness, nausea, palpitation, short breath, chest distress, dazzling/blurred vision, \\
sleeplessness (insomnia), anorexia, abdominal distension, diarrhea, constipation, cyanosis of the lips, \\
lethargy, and numbness of the extremities
\end{tabular}

\section{Experimental protocol}

We enrolled 339 healthy soldiers [means \pm standard deviations (SD): age $=24.59 \pm$ 3.27 years; height $=173.93 \pm 5.18 \mathrm{~cm}$; weight $=68.21 \pm 7.79 \mathrm{~kg}$ )] in our study that had not traveled to high altitudes in the previous 12 months. All subjects participated as volunteers and signed an informed consent form. The study was approved by the Ethics Committee of the Xinqiao Hospital, Third Military Medical University, Chongqing, China. On June 10, 2011, the subjects traveled from Xi-An (345 m in altitude) in Shaanxi Province to Golmud in Qinghai Province, China, first by train and then by bus. The subjects departed by train at 1:00 am on June 10, and arrived at Golmud settlement at 1:00 am on June 12, for a total journey time of $48 \mathrm{~h}$. The subjects were allowed an 8-h rest in XiNing, QingHai Province $(2295 \mathrm{~m})$ on the night of June 11; sufficient water was provided for the entire journey. The subjects were divided into three groups, which differed with regard to the time at which data were measured. Data were collected on June 12 for group $1(\mathrm{~N}=88)$, on June 13 for group $2(\mathrm{~N}=91)$, and on June 14 for group $3(\mathrm{~N}=160)$, such that the subjects in each group had spent one, two, or three nights, respectively, on the high plateau. The baseline characteristics for the subjects in the three groups are shown in Table 4.

Table 4. Baseline clinical characteristics for the three groups.

\begin{tabular}{lccc}
\hline & Group 1 $(\mathrm{N}=88)$ & Group 2 $(\mathrm{N}=91)$ & Group 3 $(\mathrm{N}=160)$ \\
\hline Age (years) & $25.19 \pm 4.24$ & $24.27 \pm 3.67$ & $24.44 \pm 2.25^{\#}$ \\
Height $(\mathrm{cm})$ & $173.82 \pm 5.59$ & $173.79 \pm 5.55$ & $174.08 \pm 4.75^{\#}$ \\
Weight $(\mathrm{kg})$ & $67.43 \pm 8.85$ & $68.51 \pm 7.83$ & $68.48 \pm 7.14^{\#}$ \\
BMI & $22.27 \pm 2.36$ & $22.62 \pm 1.92$ & $22.57 \pm 1.92^{\#}$ \\
Male/female & $85 / 3$ & $91 / 0$ & $160 / 0$ \\
\hline
\end{tabular}

Data are reported as frequencies or means $\pm \mathrm{SD} .{ }^{*} \mathrm{P}>0.05$.

After arrival at the campsite (3200 m altitude), located $40 \mathrm{~km}$ northwest of Golmud city, seven physicians distributed questionnaires. Blood pressure, heart rate, and oxygen 
saturation $\left(\mathrm{SpO}_{2}\right)$ levels (determined with a pocket oximeter: Nonin Onyx ${ }^{\circledR}$ 9500, Nonin Medical, Inc., USA) were also measured. All measurements were made after the subject had been seated for at least $30 \mathrm{~min}$. The local temperature was $11-24^{\circ} \mathrm{C}$, and the weather was sunny.

\section{Statistical analysis}

Data were analyzed using the SPSS 19.0 software package, and are reported as means $\pm \mathrm{SD}$. Quantitative data for the three groups were compared using one-way analysis of variance (ANOVA). Symptoms were analyzed using a non-parametric analysis and the Kruskal-Wallis test. Further analysis was carried out using the Nemenyi method. For qualitative data, the R x C method and chi-squared analyses were used. Pearson's and Spearman's correlation methods were used to analyze relationships among variables. Significance was established at $\mathrm{P}<0.05$.

\section{RESULTS}

\section{Incidences of AMS as determined by the two scoring systems}

The incidence of AMS in all 339 subjects was $17.11 \%(\mathrm{~N}=58)$ when diagnosed using the AMS-LLS. However, when the AMS-CSS was employed, the incidence was much higher, at $29.79 \%(\mathrm{~N}=101)$. There was a significant correlation between the AMS-LLS and AMS-CSS scores (Pearson's $r=0.820, \mathrm{P}<0.001$ ) (Figure 1). We found that the subjects with higher AMS-CSS scores also had higher AMS-LLS scores. All individuals diagnosed with AMS using the AMS-LLS $(\mathrm{N}=58)$ were also diagnosed with AMS using the AMSCSS. Hence, 43 subjects were diagnosed with AMS on the basis of AMS-CSS criteria that were not diagnosed with AMS using AMS-LLS criteria. These 43 subjects also suffered headache, fatigue, dizziness, insomnia, and GI symptoms $(81.4,46.5,30.2,14.0$, and $4.7 \%$, respectively). AMS was found to considerably limit the activities of the positively diagnosed patients. Two participants $(0.59 \%)$ developed HAPE, but no occurrences of HACE were reported during the first three days at high altitude. Compared with AMS-LLS, the sensitivity, specificity, positive predictive value, and negative predictive value of AMS-CSS for the diagnosis of AMS were 100, 84.7, 57.43, and 100\%, respectively.

The incidence of AMS, as diagnosed using the AMS-LLS system, was $6.82 \%(\mathrm{~N}=$ 6) in group $1,20.88 \%(\mathrm{~N}=19)$ in group 2 , and $20.63 \%(\mathrm{~N}=33)$ in group 3 . When AMSCSS criteria were used, the incidences of AMS in groups 1,2 , and 3 were $15.91 \%(\mathrm{~N}=14)$, $39.56 \%(\mathrm{~N}=36)$, and $31.88 \%(\mathrm{~N}=51)$, respectively. The grades of AMS diagnosed using the two systems are shown in Table 5.

Our findings indicate that the incidence of AMS was lowest after the first night, peaked after the second night, and then began to decrease after the third night at high altitude. Two subjects (cases 1 and 2) developed HAPE. Case 1 was given AMS-LLS and AMSCSS scores of 6 and 11, respectively, which are indicative of severe and moderate AMS, respectively. Case 2 had an AMS-LLS score of 1 and an AMS-CSS score of 2; however, this subject was diagnosed with an atrial septal defect (ASD) under the echocardiography. 


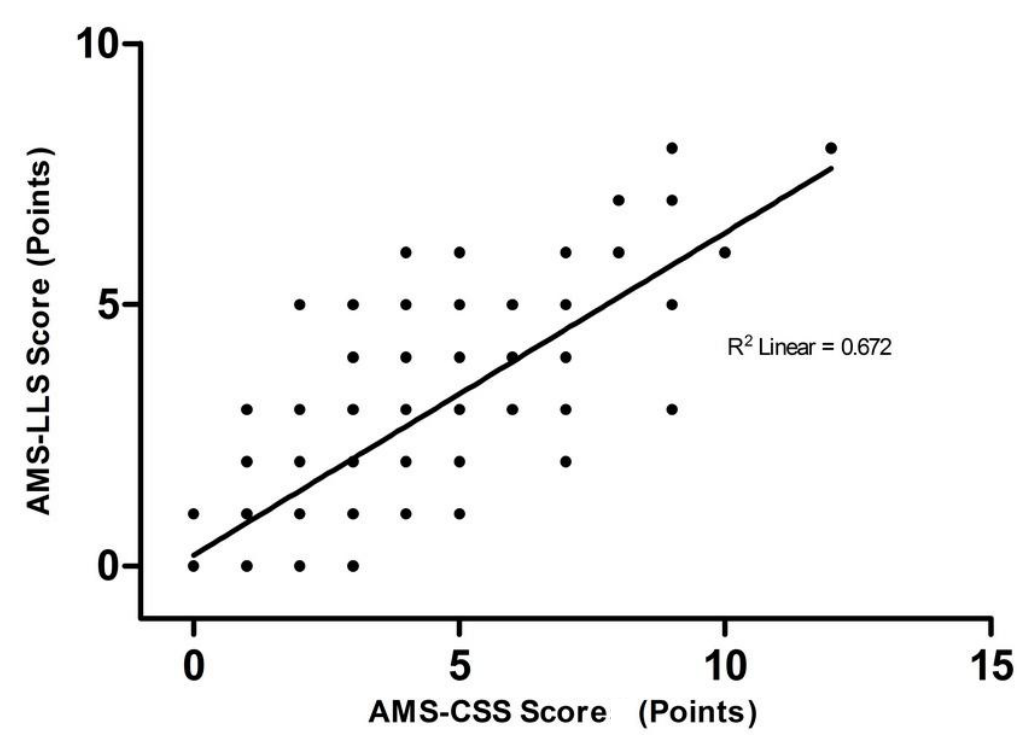

Figure 1. Correlation between acute mountain sickness-Lake Louise Scoring System (AMS-LLS) and AMSChinese Scoring System (AMS-CSS) scores. There was a significant correlation.

\begin{tabular}{|c|c|c|c|c|c|c|c|}
\hline & \multicolumn{2}{|c|}{ LLS } & \multirow[t]{2}{*}{ Sum } & \multicolumn{3}{|c|}{ CSS } & \multirow[t]{2}{*}{ Sum } \\
\hline & Mild (\%) & Severe $(\%)$ & & Mild (\%) & Moderate (\%) & Severe $(\%)$ & \\
\hline Group $1(\mathrm{~N}=88)$ & 0 & $6(6.82)$ & $6(6.82)$ & $9(10.23)$ & $5(5.68)$ & 0 & $14(15.91)$ \\
\hline Group $2(\mathrm{~N}=91)$ & $17(18.68)$ & $2(2.2)$ & $19(20.88)$ & $36(39.56)$ & 0 & 0 & $36(39.56)$ \\
\hline Group $3(N=160)$ & $22(13.75)$ & $11(6.88)$ & $33(20.63)$ & $45(28.12)$ & $6(3.75)$ & 0 & $51(31.88)$ \\
\hline
\end{tabular}

\section{Dominant symptoms}

We found that the five most common symptoms in all subjects were decreased exercise tolerance $(61.7 \%)$, fatigue $(49.0 \%)$, dizziness $(28.9 \%)$, chest distress $(28.3 \%)$, and headache (27.4\%) (Figure 2). Headache was not the most common symptom, but was ranked eighth $(12.7 \%)$ in group 1 , fourth $(37.4 \%)$ in group 2 , and third $(29.4 \%)$ in group 3 . The incidence of headache differed significantly among the three groups $(\mathrm{P}<0.05)$. Further analysis showed that headache was not related to $\mathrm{SpO}_{2}$ levels $(\mathrm{P}=0.35)$. Decreased activity and fatigue were the most common complaints in all groups. Vomiting, which is considered to be a very important symptom in AMS-CSS, was not reported in our study. We compared the order of symptoms by using five AMS-LLS parameters. The order of the symptoms was the same in the two-system-positive subjects: headache, fatigue, dizziness, insomnia, and GI symptoms, which all gradually reduced (Table 6).

Comparison of the symptoms between groups revealed that there were significant differences in the incidences of headache and fatigue $(\mathrm{P}=0.004$ and $\mathrm{P}<0.001$, respectively). Further analysis showed that the incidence of headache in group 1 was lower than in groups 2 
$(\mathrm{P}=0.0063)$ and $3(\mathrm{P}=0.048)$, whereas no difference was observed between groups 2 and 3 $(\mathrm{P}=0.5251)$. The incidence of fatigue in group 1 was also lower than in groups $2(\mathrm{P}=0.0019)$ and $3(\mathrm{P}<0.001)$, with no significant difference between groups 2 and $3(\mathrm{P}=0.7023)$. The incidences of other symptoms, including GI upset, abdominal distention, diarrhea, and constipation, were not significantly different between the three groups.

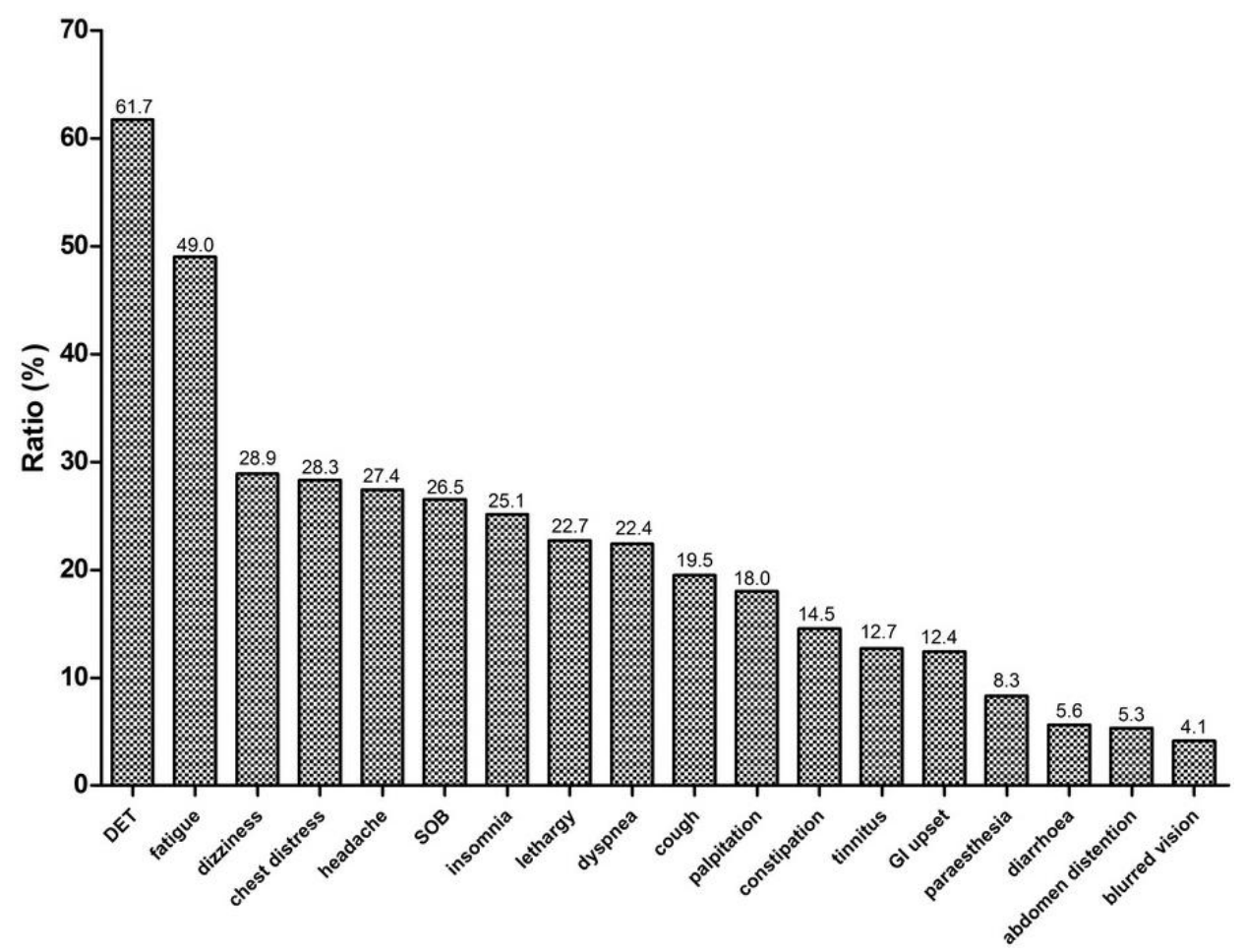

Figure 2. Symptoms in all cases at $3200 \mathrm{~m}$ in altitude $(\mathrm{N}=339)$. DET $=$ decreased exercise tolerance; $\mathrm{SOB}=$ shortness of breath; GI upset $=$ gastrointestinal upset.

Table 6. Order of symptoms in the two-system positive-subjects.

\begin{tabular}{lcc}
\hline & AMS-CSS positive $(\mathrm{N}=101, \%)$ & AMS-LLS positive $(\mathrm{N}=58, \%)$ \\
\hline Headache & 89.1 & 100.0 \\
Fatigue & 73.3 & 93.1 \\
Dizziness & 50.5 & 65.5 \\
Insomnia & 35.6 & 51.7 \\
GI symptom & 24.8 & 39.7 \\
\hline
\end{tabular}

AMS-CSS = acute mountain sickness-Chinese Scoring System; AMS-LLS = AMS-Lake Louise Scoring System.

\section{Relationship between vital signs and the two scoring systems}

We found that neither the AMS-LLS nor AMS-CSS scores were related with $\mathrm{SpO}_{2}$ levels ( $\mathrm{P}=0.773$ and $\mathrm{P}=0.882$, respectively). Furthermore, there were no significant differences in systolic blood pressure $(\mathrm{F}=0.481, \mathrm{P}=0.619)$, diastolic blood pressure $(\mathrm{F}=0.051, \mathrm{P}$ 
$=0.950)$, heart rate $(\mathrm{F}=1.186, \mathrm{P}=0.307)$, and $\mathrm{SpO}_{2}$ levels $(\mathrm{F}=0.203, \mathrm{P}=0.307)$ among the three groups. These vital signs remained relatively unchanged during the first three days after ascent to high altitude (Figure 3). We also found no significant differences in vital signs between non-AMS and AMS subjects, whether diagnosed by using the AMS-CSS or the AMSLLS $(\mathrm{P}>0.05)$.

A

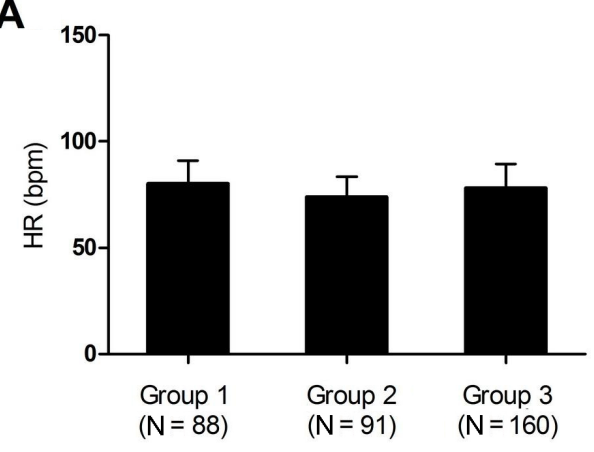

C

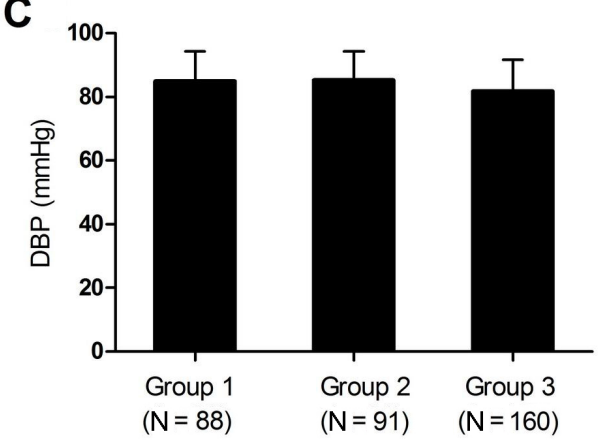

B

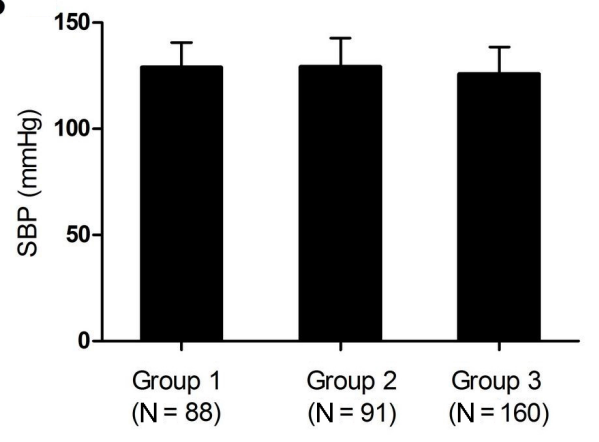

D

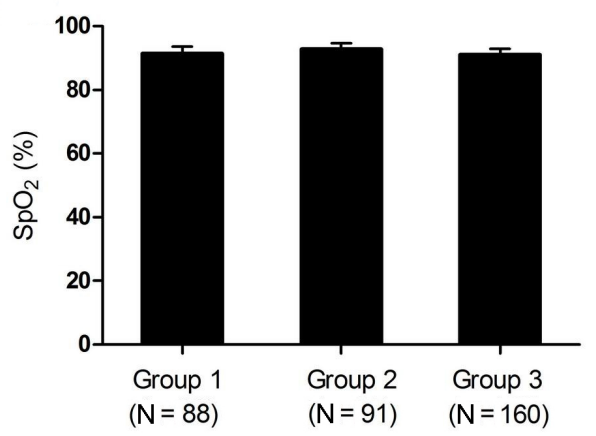

Figure 3. Vital signs in the three groups. Vital signs among the three groups did not differ significantly . A. HR $=$ heart ratio $(\mathrm{P}=0.307)$; $\mathbf{B}$. $\mathrm{SBP}=$ systolic blood pressure $(\mathrm{P}=0.619) ; \mathbf{C}$. $\mathrm{DBP}=$ diastolic blood pressure $(\mathrm{P}=$ 0.950); D. $\mathrm{SpO}_{2}=$ oxygen saturation $(\mathrm{P}=0.307)$.

\section{DISCUSSION}

To the best of our knowledge, this is the first study to show that the incidences of AMS in the same cohort of subjects were significantly different when determined using the AMS-LLS and AMS-CSS. Our findings demonstrate that a rapid ascent to $3200 \mathrm{~m}$ results in a higher incidence of AMS as defined by the AMS-CSS than that determined by the AMS-LLS questionnaire, although a correlation between the two systems was evident. We found that all of AMS patients that were diagnosed using the AMS-LLS were also identified as having AMS using the AMS-CSS.

The reported incidences of AMS, according to the AMS-LLS, have varied substantially between studies because of the different protocols and altitudes used. Vardy et al. (2006) found that the incidence of AMS was 0 at 2500-3000 $\mathrm{m}$ and was $10 \%$ at $3000-4000 \mathrm{~m}$. Karinen et 
al. (2010) reported an incidence of $10 \%$ at $3500 \mathrm{~m}$ and $21 \%$ at $4300 \mathrm{~m}$, whereas Newcomb et al. (2011) found that $21 \%$ of Caucasian hikers suffered from AMS at $4470 \mathrm{~m}$. Using subjects with the same ethnic background as those of our study, researchers from Taiwan found that the incidence of AMS at Jade mountain (3950 m) was approximately 36\% (Wang et al., 2010), a value significantly higher than that observed in the present study $(17.11 \%$ at $3200 \mathrm{~m})$. There are several possible explanations for the discrepancy between our data and those of Wang et al. First, the altitude in the latter study was higher than that in ours (3950 vs $3200 \mathrm{~m})$. Second, the subjects in their study ascended at a faster rate than did our volunteers (20.54 vs $48 \mathrm{~h})$. Third, their participants were hikers, whereas ours were young soldiers who had strenuously trained at sea level (both aerobic and anaerobic exercise), and therefore might have been more able to tolerate hypoxia at high altitude.

Using the AMS-CSS, the incidence of AMS in Chinese male soldiers at $3600 \mathrm{~m}$ was reported to be $57.2 \%$ (Ren et al., 2010), a value higher than that found in the present study (29.79\%). Ren et al. (2010) subjects traveled to Tibet (3600 m) by air, whereas our participants traveled by train and bus. In addition, our camp was located at $\sim 3200 \mathrm{~m}$ rather than at $3600 \mathrm{~m}$. It is well known that the incidence of AMS is influenced by the method of travel and the rate of ascent to high altitude (Schneider et al., 2002). Furthermore, the study by Ren et al. (2010) included lip color ('bluish') as one of the parameters used to diagnose AMS, and this symptom was present in $95.31 \%$ of their participants. We did not use this parameter in our AMS-CSS, as we believe that this is not a specific sign of AMS at high altitude, based on the results of Ren et al. Our decision not to use this parameter may have decreased the total AMS-CSS score, and hence the incidence of AMS, compared with observations of Ren et al.

The AMS-CSS considers vomiting to be an important symptom of AMS, which is weighted as heavily as headache, and also classifies subjects according to the vomiting frequency and administration of antiemetic medicines. Subjects are diagnosed to have AMS if they present either vomiting or headache as a symptom, or have a total AMS-CSS score greater than or equal to 5 points. Some Chinese researchers have suggested that the CSS is more objective than the LLS. In the AMS-CSS, the headache symptom is graded by the effect of oral analgesia drug, and vomiting is graded by the frequency of the symptom. The AMS-CSS divides vomiting 1-2, 3-4, and $\geq 5$ times per day into mild, moderate, and severe categories, respectively. By contrast, in the AMS-LLS, the symptom grading relies only on the participants' own subjective feeling. This is why many Chinese researchers believe that the AMS-CSS is more objective than the AMS-LLS. However, there was no vomiting case reported in our subjects in the present study. In Ren et al. (2010), in which AMS was evaluated with a more rapid ascent and at higher altitude, only $12 \%$ of the subjects suffered vomiting symptoms. Therefore, vomiting might not be as relevant to AMS as previously thought, or at least not at a high altitude of $3200 \mathrm{~m}$. Therefore, the role of vomiting in the AMS-CSS is worthy of further study. Compared with the AMS-CSS, it may be more reasonable for the AMS-LLS to group all GI symptoms as a single parameter.

It is worth noting that headache is not an essential element in the AMS-CSS, whereas it is considered to be an essential symptom in the AMS-LLS. Our data demonstrated that headache was not the most dominant symptom after ascent to $3200 \mathrm{~m}$, especially during the first three days. Since many subjects did not present headache in our study, they did not meet the diagnosis of AMS based on the AMS-LLS, which possibly contributed to the lower incidence of AMS diagnosed with the AMS-LLS as compared with the AMS-CSS. However, most of 
the subjects who were positive based on the AMS-CSS but negative in the AMS-LLS suffered from headache, fatigue, dizziness, and other symptoms. We found that all subjects diagnosed with AMS using the AMS-LLS were also identified using the AMS-CSS. This suggests that individuals positively diagnosed with the AMS-LLS are also included within the AMS-CSS criteria. It is important that we consider not only subjects diagnosed by both sets of criteria, but also those identified by the AMS-CSS and not the AMS-LLS. If the AMS-LLS alone is used to diagnose AMS, this may exclude some individuals without headache but with the potential to develop HAPE or HACE. Some patients can develop HAPE directly. Our results showed that in HAPE patients without ASD, the AMS scores were high, whereas in HAPE patients with ASD, the AMS scores were low using both systems. It is suggested that if a person with ASD has a higher AMS score, more attention should be paid to prevent HAPE.

There is no gold standard based on pathology and imaging for the diagnosis of AMS and grading of its severity; instead, its diagnosis relies on a scoring system that depends only on the symptoms present. As a result, we can only take the AMS-LLS as the gold standard when calculating the sensitivity of the AMS-CSS. We found that the sensitivity of the AMSCSS was very high, and was equal to that of the AMS-LLS. Compared with the AMS-CSS, the AMS-LLS is a simpler diagnostic scheme that includes only five parameters, and hence is easier to commit to memory and use as a survey. In contrast, the AMS-CSS has many parameters and therefore it might be more difficult to work with in the field.

We here showed that the incidence of AMS was lowest after the first night and highest after the second night at high altitude, and then tended to decrease after the third night. Our results are in accordance with those of previous studies that reported that AMS often appeared 6-12 $\mathrm{h}$ after the subject's arrival in a high-altitude environment, and usually resolved within 1-3 days (Imray et al., 2010). It is well known that AMS is an acute response to hypoxia. It is likely that the maximum response to hypoxia occurs after the second night following ascent to $3200 \mathrm{~m}$. Once the body has partially compensated for the hypoxia, the symptoms improve after the third night at high altitude.

$\mathrm{SpO}_{2}$ is an important index of hypoxia. We found no relationship between the incidence of AMS and the $\mathrm{SpO}_{2}$ level, at least not at an altitude of $3200 \mathrm{~m}$, in our subjects and protocol, irrespective of whether the AMS-CSS or AMS-LLS was used. This is in agreement with the findings of O'Connor et al. (2004). Similarly, Karinen et al. (2010) found that at 3000 $\mathrm{m}, \mathrm{SpO}_{2}$ did not differ between the AMS and non-AMS groups. However, the authors found that reduced $\mathrm{SpO}_{2}$ levels at rest and during exercise, measured at altitudes of 3500 and 4300 $\mathrm{m}$, seemed to predict impending AMS at altitudes of 4300 and $5300 \mathrm{~m}$ (Karinen et al., 2010). Their results suggest that $\mathrm{SpO}_{2}$ may be a useful marker for predicting AMS at altitudes of 3500 $\mathrm{m}$ or higher.

\section{Limitations}

Although our study included a large number of participants, it nonetheless has a number of limitations, including the short duration of the study and the lack of data with which to compare the effects of different altitudes and varying rates of ascent. Our subjects were all young adults who were part of a well screened, medically checked, and regularly exercising population. Therefore, we are unable to extend our findings to other groups, such as the elderly or adolescents. The moderate elevation and 48 -h ascent exert a fairly mild altitude stress 
as demonstrated by the lack of moderate or severe symptoms. The prevalence of HAPE and HACE were also so low that we were unable to determine which of the two systems might prove to be a better predictor of the incidence of these conditions.

\section{CONCLUSION}

In summary, we found that the two systems used for the diagnosis of AMS have their own advantages and disadvantages. The AMS-CSS is similar to the AMS-LLS with some different details. Both provide useful diagnostic criteria that have been used and, to some extent, validated for more than 15 years, but both are also more subjective than objective. Further experimental evidence and clinical trials are warranted to learn more about AMS and to identify the best criteria for diagnosis. Headache was not the dominant symptom in our study. Furthermore, the vomiting symptom might be not as important as previously thought in the AMS-CSS. In addition, $\mathrm{SpO}_{2}$ levels did not correlate with AMS.

\section{ACKNOWLEDGMENTS}

Research supported by the Special Health Research Project of the Ministry of Health of the People's Republic of China (\#201002012). We would like to thank Xin Du, M.D., Cardiology Department, Tianjin Medical University General Hospital, for advice on the manuscript. We also express sincere gratitude to Zhe-Xue Qin, M.D., and Qing Wang, M.D., Institute of Cardiovascular Diseases of PLA, Xinqiao Hospital, Third Military Medical University, for helpful discussions.

\section{REFERENCES}

Imray C, Wright A, Subudhi A and Roach R (2010). Acute mountain sickness: pathophysiology, prevention, and treatment. Prog. Cardiovasc. Dis. 52: 467-484.

Jiao XJ, Fang T, Fan HJ and Hou SK (2010). Physiological adaptability of members of Chinese National Earthquake Disaster Emergency Rescue Team (CNEDERT) in altitude of Yushu area of Qinghai province and prevention of acute mountain sickness. Chin. J. Emerg. Med. 19: 829-833.

Karinen HM, Peltonen JE, Kähönen M and Tikkanen HO (2010). Prediction of acute mountain sickness by monitoring arterial oxygen saturation during ascent. High Alt. Med. Biol. 11: 325-332.

Kayser B, Aliverti A, Pellegrino R, Dellaca R, et al. (2010). Comparison of a visual analogue scale and Lake Louise symptom scores for acute mountain sickness. High Alt. Med. Biol. 11: 69-72.

Luks AM, McIntosh SE, Grissom CK, Auerbach PS, et al. (2010). Wilderness Medical Society consensus guidelines for the prevention and treatment of acute altitude illness. Wilderness Environ. Med. 21: 146-155.

Newcomb L, Sherpa C, Nickol A and Windsor J (2011). A comparison of the incidence and understanding of altitude illness between porters and trekkers in the Solu Khumbu Region of Nepal. Wilderness Environ. Med. 22: 197-201.

O'Connor T, Dubowitz G and Bickler PE (2004). Pulse oximetry in the diagnosis of acute mountain sickness. High Alt. Med. Biol. 5: 341-348.

Ren Y, Fu Z, Shen W, Jiang P, et al. (2010). Incidence of high altitude illnesses among unacclimatized persons who acutely ascended to Tibet. High Alt. Med. Biol. 11: 39-42.

Roach RC, Bärtsch P, Hackett PH and Oelz O (1993). The Lake Louise Acute Mountain Sickness Scoring System. In: Hypoxis and mountain medicine (Lake Louise AMS Scoring Committee, eds.). Queen City Printer, Burlington, 272-274.

Roach R, Kayser B and Hackett P (2011). Pro: Headache should be a required symptom for the diagnosis of acute mountain sickness. High Alt. Med. Biol. 12: 21-22.

Sanchez del Rio M and Moskowitz MA (1999). High altitude headache. Lessons from headaches at sea level. Adv. Exp. Med. Biol. 474: 145-153. 
Schneider M, Bernasch D, Weymann J, Holle R, et al. (2002). Acute mountain sickness: influence of susceptibility, preexposure, and ascent rate. Med. Sci. Sports Exerc. 34: 1886-1891.

Third Ad Hoc Committee on High Altitude Illnesses of Chinese Medical Association (1996). Consensus statement for the criteria for the denomination, classification and diagnosis of high altitude illnesses in China. Tibetan Med. J. 17: 1-3.

Vardy J, Vardy J and Judge K (2006). Acute mountain sickness and ascent rates in trekkers above $2500 \mathrm{~m}$ in the Nepali Himalaya. Aviat. Space Environ. Med. 77: 742-744.

Wang SH, Chen YC, Kao WF, Lin YJ, et al. (2010). Epidemiology of acute mountain sickness on Jade Mountain, Taiwan: an annual prospective observational study. High Alt. Med. Biol. 11: 43-49.

West JB (2010). English translation of "Nomenclature, classification, and diagnostic criteria of high altitude disease in China". High Alt. Med. Biol. 11: 169-172.

West JB (2011). Con: Headache should not be a required symptom for the diagnosis of acute mountain sickness. High Alt. Med. Biol. 12: 23-25.

Wu T (2011). Mountain rescue: the highest earthquake in Yushu. High Alt. Med. Biol. 12: 93-95.

Wu TY, Ding SQ, Liu JL, Yu MT, et al. (2009). Reduced incidence and severity of acute mountain sickness in QinghaiTibet railroad construction workers after repeated 7-month exposures despite 5-month low altitude periods. High Alt. Med. Biol. 10: 221-232. 